\title{
Choroidal Volume Variations During Childhood
}

\author{
Chiara Mapelli, ${ }^{1,2}$ Laura Dell'Arti, ${ }^{1}$ Giulio Barteselli, ${ }^{1}$ Silvia Osnaghi, ${ }^{2}$ Elena Tabacchi, ${ }^{2}$ \\ Michele Clerici, ${ }^{1}$ Roberto Ratiglia, ${ }^{1,2}$ and Francesco Viola ${ }^{1,2}$ \\ ${ }^{1}$ University of Milan, Department of Clinical Sciences and Community Health, Milan, Italy \\ ${ }^{2}$ Ophthalmological Unit, Ca' Granda Foundation-Ospedale Maggiore Policlinico, Milan, Italy
}

Correspondence: Francesco Viola, U.O. Oculistica, Dipartimento di Scienze Cliniche e di Comunità, Fondazione IRCCS Ca' Granda-Ospedale Maggiore Policlinico, University of Milan, Milan, Italy;

francesco.viola@unimi.it

Submitted: July 8, 2013

Accepted: September 16, 2013

Citation: Mapelli C, Dell'Arti L, Barteselli $G$, et al. Choroidal volume variations during childhood. Invest Ophthalmol Vis Sci. 2013;54:68416845. DOI:10.1167/iovs.13-12761
Punpose. We analyzed choroidal volume (CV) variations during childhood using enhanced depth imaging optical coherence tomography, and evaluated its association with age, axial length (AXL), sex, weight, and height.

Methods. Imaging studies of the right eyes of 52 healthy children were reviewed and included in this study. Subjects underwent a complete ocular examination and AXL measurement, as well as a raster macular scan using the Heidelberg Spectralis device. The choroid was segmented manually.

REsults. Subjects included 21 males and 31 females, with mean age of 9 years (range, 2-17 years) and mean AXL of $22.8 \pm 0.98 \mathrm{~mm}$. Mean CV was $0.263 \pm 0.068 \mathrm{~mm}^{3}$ for the foveal circle and $8.545 \pm 1.822 \mathrm{~mm}^{3}$ for the total Early Treatment of Diabetic Retinopathy Study (ETDRS) grid. The CV of the nasal quadrant was significantly lower than all others $(P<$ 0.001). Total and foveal CV showed significant negative correlation with AXL after adjustment for age $(P<0.001)$, and significant positive correlation with age after adjustment for AXL $(P<$ $0.001)$. Total CV was correlated significantly with sex after adjusting for AXL $(P=0.01)$, while no correlations were found between total $\mathrm{CV}$ and height or weight. The $\mathrm{CV}$ increased by $0.214 \mathrm{~mm}^{3}$ (2.5\%) for every year, and decreased by $1.0 \mathrm{~mm}^{3}(11.7 \%)$ for every millimeter of axial length. Regression analysis confirmed a trend of higher $\mathrm{CV}$ in females than in males $(P=$ 0.056).

Conclusions. The CV increases with age during childhood, but decreases with AXL. This finding supports the hypothesis that the choroid grows progressively during childhood. Intersexual differences of CV also may be present.

Keywords: choroidal volume, choroidal thickness, enhanced depth imaging, optical coherence tomography
$T$ he choroid is a vascular structure with multiple functions in the eye, including metabolic support of the RPE, and the retina and blood supply to the outer retinal layers. ${ }^{1,2}$ The choroid also is a primary site of involvement in various chorioretinal diseases, such as central serous chorioretinopathy, choroidal neovascularization, polypoidal choroidal vasculopathy, Vogt-Koyanagi-Harada (VKH) syndrome, and other chorioretinal inflammatory disorders. ${ }^{3}$ Therefore, evaluation of the choroid would be helpful to understand the pathophysiology, and improve diagnosis and management of chorioretinal disorders.

The enhanced depth imaging (EDI) on spectral-domain optical coherence tomography (SD-OCT) has become a useful tool to perform in vivo quantitative assessment of the choroidal structure. ${ }^{4}$ Using EDI-OCT, the chorioscleral junction can be visualized easily, and its distal margin can be used to measure choroidal thickness. ${ }^{5}$ The most commonly used method to measure the choroid on EDI-OCT scans is the bidimensional manual assessment of choroidal thickness in the fovea and a few perifoveal locations. ${ }^{6}$ However, measuring thickness only at selected points of the choroid may not describe the anatomy of the choroid comprehensively, since the irregularity of the inner chorioscleral border may influence the measurements. ${ }^{7}$ To overcome this problem, and to obtain a better picture of the state of the posterior choroid, other investigators have devised an innovative method of 3-dimensional choroidal volume measurement using the Heidelberg Spectralis instrument (Heidelberg Engineering, Heidelberg, Germany). ${ }^{8,9}$ Briefly, after performing manual segmentation of the choroid on each B-scan of a 31-scan raster protocol centered on the fovea, the built-in software for retinal volume analysis of the device provides automatic measurement of the choroidal volume in the 6-mm diameter Early Treatment Diabetic Retinopathy Study (ETDRS) grid. Choroidal volume variations according to age, axial length (AXL), and sex subsequently have been reported in healthy subjects with a mean age of 50 years; age and axial length are correlated inversely with choroidal volume, and differences due to subjects' sex also may be present. ${ }^{4}$

So far, the development of the choroid in children has received little attention. In addition, results about the relationship between choroidal thickness and age in children are not consistent in the published literature, ${ }^{10-12}$ probably because the choroidal thickness was measured at few sampling points. Since the choroid is a 3-dimensional structure, a volumetric analysis would be helpful to clarify the development of the choroid in children. Therefore, in the present study we aimed to measure the choroidal volume at the posterior pole in healthy children, and to assess its relationship with multiple variables, including age, AXL, sex, weight, and height. 


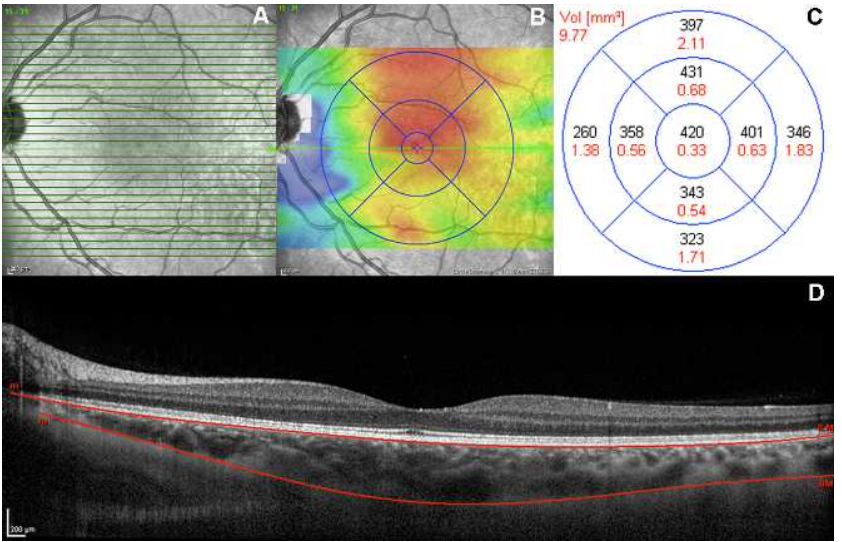

Figure 1. Choroidal volume scanning protocol and image analysis. (A) A total of 31 high-resolution B-scans centered at the fovea, associated with a simultaneous near-infrared image. (B) Color-coded choroidal thickness map. (C) Choroidal volume of each subfield of the ETDRS grid. (D) Manual segmentation of the choroid.

\section{Methods}

\section{Study Population}

A retrospective observational study was performed in a tertiary center in Milan (Ophthalmological Unit, Ca' Granda Foundation-Ospedale Maggiore Policlinico, Milan, Italy). The research adhered to the tenets of the Declaration of Helsinki, and approval by the investigational review board (IRB) of the $\mathrm{Ca}$ ' Granda Foundation-Ospedale Maggiore Policlinico was obtained. Informed consent was obtained from the parents of the subjects after IRB approval.

Charts and images of 52 right eyes of 52 healthy children who underwent OCT as part of their visit for screening purposes over a 6-month period (October 2012 through March 2013) were reviewed. We included children between 2 and 17 years of age, with no known systemic or ocular disease. We specifically excluded children born prematurely, as well as cases presenting with maculopathy, congenital glaucoma or cataract, amblyopia, strabismus, refractive error of more than 2 diopters (D), or best-corrected visual acuity (BCVA) less than 0.8 Snellen equivalent. All patients underwent a complete ocular examination, including BCVA measurement, slit-lamp examination, and AXL measurement (IOL Master; Carl Zeiss Meditec, Jena, Germany). Indirect ophthalmoscopy also was performed after pupillary dilation.

\section{OCT Imaging}

A raster scan of the macula using an SD-OCT device in EDI mode (Heidelberg Spectralis; Heidelberg Engineering) was used to study the choroid, with a protocol used in previously reported studies (Fig. 1). 4,8,9 The scanning protocol included 31 high-resolution B-scans; each scan was approximately 9.3 $\mathrm{mm}$ in length and spaced $240 \mu \mathrm{m}$ apart. All 31 B-scans were acquired in a continuous, automated sequence and covered a $30^{\circ} \times 25^{\circ}$ area centered on the fovea. A minimum of 10 frames were averaged automatically and used to obtain a good quality choroidal image using the built-in TruTrack Active Eye Tracking software (Heidelberg Engineering). One trained physician manually performed the choroidal segmentation using a previously described well-reproducible method. ${ }^{8}$ To ensure reproducibility of the choroidal volume measurements, a second trained physician repeated the segmentation of the choroid from images of 20 randomly selected eyes. The colorcoded choroidal volume map centered on the fovea was
TABLE 1. Baseline Characteristics of the Sample

\begin{tabular}{ll}
\hline $\begin{array}{l}\text { Number of eyes } \\
\text { Sex }\end{array}$ & 52 \\
$\quad$ Male & $21(40.4 \%)$ \\
$\quad$ Female & $31(59.6 \%)$ \\
Age, $\mathrm{y}$ & \\
$\quad$ Range & $2-17$ \\
$\quad$ Mean \pm SD & $9.3 \pm 3.7$ \\
Axial length, mm & \\
$\quad$ Range & $20.85-24.97$ \\
Mean \pm SD & $22.8 \pm 0.98$ \\
Weight, kg & \\
$\quad$ Range & $17-64$ \\
Mean \pm SD & $38 \pm 14$ \\
Height, cm & \\
Range & \\
Mean \pm SD & $110-180$ \\
\hline
\end{tabular}

generated automatically by the built-in software of the device by applying the ETDRS grid, and measurements of the choroidal volume were recorded (Fig. 1). The ETDRS grid divides the macula into 3 concentric rings (center, inner, and outer), with the inner ring measuring 1 to $3 \mathrm{~mm}$ and the outer ring measuring 3 to $6 \mathrm{~mm}$ of diameter (referring to a ring with a diameter of $1 \mathrm{~mm}$ centered on the fovea). The grid further divides inner and outer rings into 4 quadrants (superior, inferior, temporal, and nasal).

\section{Statistical Analysis}

Statistical analysis was performed using the Statistical Package for the Social Sciences version 20 (SPSS, Inc., Chicago, IL). All values are presented as mean \pm SD. Post hoc Tukey's analysis was used to compare the choroidal volume at different ETDRS quadrants. Multiple linear regression analysis was used to evaluate the correlation between the choroidal volume and independent variables, including age, AXL, sex, weight, and height of the subjects. The agreement between interobserver measurements was assessed using the intraclass correlation coefficient. $P \pm 0.05$ was considered to be statistically significant.

\section{Results}

We included 52 right eyes of 21 males and 31 females, with a mean age of 9 years (range, 2-17 years). The baseline characteristics of the subjects are reported in Table 1. All subjects were Caucasian.

Reproducibility of choroidal volume measurements between the two physicians was excellent, with an intraclass correlation coefficient of 0.96 . Table 2 shows the mean choroidal volume in each area of the ETDRS map. Mean choroidal volume was $0.263 \pm 0.068 \mathrm{~mm}^{3}$ for the foveal circle and $8.545 \pm 1.822 \mathrm{~mm}^{3}$ for the total ETDRS map. Mean choroidal volume was $1.686 \pm 0.469,2.178 \pm 0.468,2.271 \pm$ 0.460 , and $2.146 \pm 0.477 \mathrm{~mm}^{3}$ for the nasal, superior, temporal, and inferior quadrants, respectively. Choroidal volume of the nasal quadrant was significantly lower than all the others $(P<0.001)$. No differences in choroidal volume were found among the superior, temporal, and inferior quadrants. Total and foveal choroidal volume showed significant positive correlation with age (Figs. $2 \mathrm{~A}-\mathrm{C}$ ) after adjustment for AXL $(P<0.001)$, and significant negative correlation with 
TABLE 2. Choroidal Volume Measurements $\left(\mathrm{mm}^{3}\right)$ for Each Subfield of the ETDRS Grid Centered on the Macula

\begin{tabular}{|c|c|c|c|}
\hline Subfield & Mean & SD & Median \\
\hline Fovea & 0.263 & 0.068 & 0.255 \\
\hline Superior & 2.178 & 0.468 & 2.155 \\
\hline Nasal & $1.686^{*}$ & 0.469 & 1.675 \\
\hline Inferior & 2.146 & 0.477 & 2.040 \\
\hline Temporal & 2.271 & 0.460 & 2.205 \\
\hline Total & 8.545 & 1.823 & 8.240 \\
\hline
\end{tabular}

AXL (Figs. 2D-F) after adjustment for age $(P<0.001)$. Total choroidal volume was correlated significantly with sex after adjusting for AXL $(P=0.01)$. No correlations were found between total choroidal volume and height or weight of the subjects.

Table 3 shows the results of the multiple linear regression analysis for mean choroidal volume of the ETDRS grid. A stepwise method was used to determine the most unexpected factors. The model determined by age, AXL, and sex had the best regression, with a coefficient of determination $\left(R^{2}\right)$ of 0.359 for the total choroidal volume. Figure 3 shows a significant negative correlation of the total choroidal volume in the ETDRS map with axial length $\left(P<0.001, R^{2}=0.107\right)$, and a significant positive correlation with age $\left(P=0.003, R^{2}=\right.$ $0.101)$. Univariate regression analysis showed an approximate decrease in choroidal volume of $1.0 \mathrm{~mm}^{3}$ (11.7\%) for every millimeter of AXL, and an approximate increase in choroidal volume of $0.214 \mathrm{~mm}^{3}$ (2.5\%) for every year. Regression analysis confirmed a trend of higher choroidal volumes in females than in males $(P=0.056)$.

\section{Discussion}

With the recent development of the EDI technique on SD-OCT devices, in vivo assessment of the choroid has become an area of interest. With this technique, it is possible not only to measure the thickness of the choroid at a few sampling points, but also to calculate the volume of the whole macular choroid. What emerges from the literature is that choroidal thickness and volume in healthy adults decrease with age and with the severity of myopia. ${ }^{4,13-16}$ Intersexual differences also have been reported. ${ }^{4,17}$ However, all these studies focused on adults; data on choroidal thickness in children are scarce and discordant. ${ }^{10-12}$

Our study was designed to analyze the relationship between the 3-dimensional structure of the choroid and a variety of variables in children under 18 years old. The most striking finding is that the volume of the choroid increases during childhood, while it is well known that it decreases with age in adulthood. ${ }^{4,15}$ This suggests that the choroid grows as the other ocular structures do during childhood; indeed, during this period the lens thickens progressively, and the vitreous cavity elongates. So far, only a few reports have investigated the growth of the choroid during childhood, with inconsistent results. Some investigators described that the thickness of the choroid increases significantly from early childhood to adolescence, ${ }^{11,12}$ while others found that the choroid in children is not thicker than that of healthy adults, except for the temporal side of the fovea. ${ }^{10}$ An explanation for these discordant results may be the method that the investigators used to measure the choroidal thickness manually: Measurements taken at multiple single points could be misleading in the overall assessment of the choroidal anatomy, especially
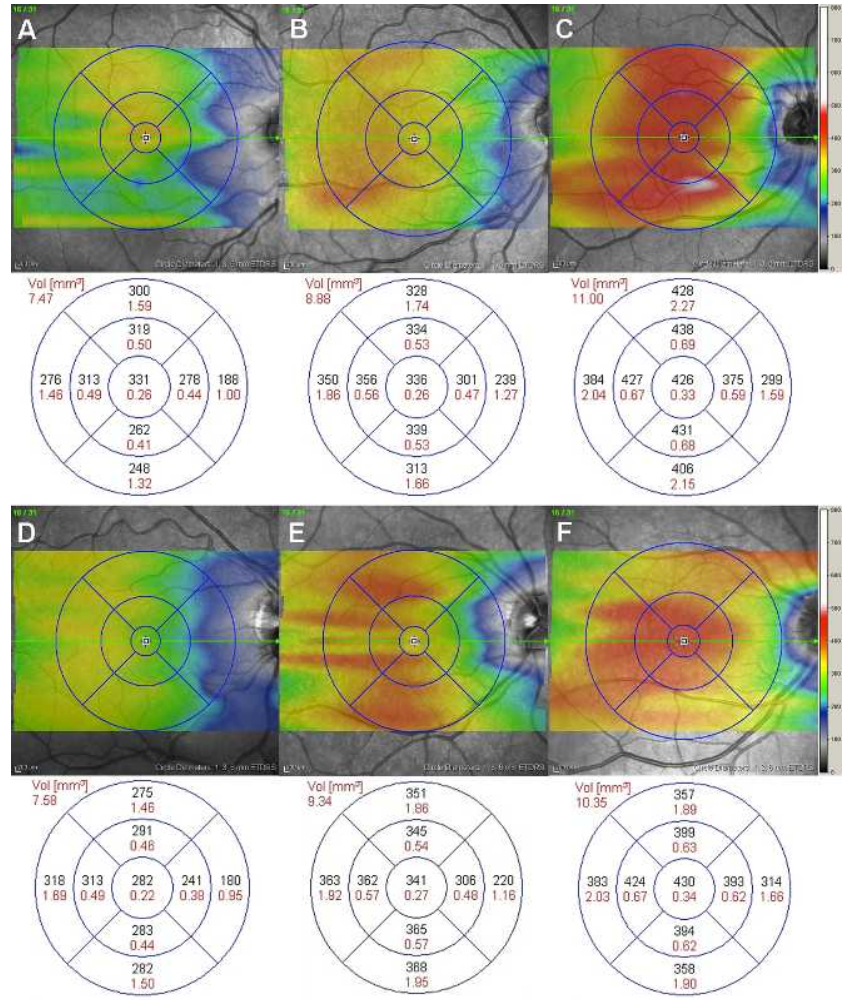

Figure 2. Effect of age (A-C) and axial length (D-F) on the colorcoded choroidal thickness map of the ETDRS grid. (A) Right eye of a 17-year-old boy; total choroidal volume is $7.47 \mathrm{~mm}^{3}$. (B) Right eye of a 10-year-old boy; total choroidal volume is $8.88 \mathrm{~mm}^{3}$. (C) Right eye of a 4-year-old girl; total choroidal volume is $11.00 \mathrm{~mm}^{3}$. (D) Myopic eye (axial length $=24.63 \mathrm{~mm}$ ); total choroidal volume is $7.58 \mathrm{~mm}^{3}$. (E) Emmetropic eye (axial length $=23.53 \mathrm{~mm}$ ); total choroidal volume is $9.34 \mathrm{~mm}^{3}$. (F) Hyperopic eye (axial length $=21.26 \mathrm{~mm}$ ); total choroidal volume is $10.35 \mathrm{~mm}^{3}$.

because the chorioscleral border may be irregular. ${ }^{7}$ Since the choroid is a 3-dimensional structure, we believed that a volumetric analysis of the choroid may reflect more accurately the anatomy of this highly vascular tissue than a simple bidimensional measurement. The 3-dimensional assessment of the choroid that we performed in our study supported the finding that this highly vascular tissue does get thicker during childhood, and then reaches a plateau of the growth in early adulthood. ${ }^{11}$ In addition, in our study we calculated an average rate of growth of the macular choroidal volume of $0.214 \mathrm{~mm}^{3}$ per year. Considering that in adults an approximate decrease in volume of $0.54 \mathrm{~mm}^{3}$ every 10 years has been shown previously using the same method of choroidal volume measurement, ${ }^{4}{ }^{\text {it's }}$ likely that the choroid grows faster during early childhood than it declines after the peak in early adulthood. Read et al. suggested that by the age of 12 years the choroidal thickness approaches adult values, and reaches a peak between 10 and 20 years of age. ${ }^{11}$ Further longitudinal prospective studies may clarify the exact age corresponding to the peak of the development of the choroid.

In our study we also investigated the topographic distribution of choroidal volume in the macula. The nasal quadrant of the ETDRS grid showed the lowest choroidal volume among all the quadrants, as also already reported in adults. ${ }^{4}$ Previous investigators also have reported a thinning of the nasal region of the macula in children, ${ }^{10-12}$ but their results are not completely comparable with our findings, since none of these investigators measured the volume of the choroid. Ruiz- 
TABLE 3. Multiple Linear Regression Analysis for Choroidal Volume of the Macula by Age, Axial Length, and Sex

\begin{tabular}{lrccr}
\hline \multicolumn{1}{c}{ Factor } & Coefficient & SE & $\boldsymbol{R}^{2}$ & \multicolumn{1}{c}{$\boldsymbol{P}$} \\
\hline Intercept & 28.090 & 5.617 & 0.359 & $<0.001$ \\
Age & 0.214 & 0.068 & 0.359 & 0.003 \\
Sex & 0.875 & 0.446 & 0.359 & 0.056 \\
Axial length & -1.005 & 0.252 & 0.359 & $<0.001$ \\
\hline
\end{tabular}

Moreno et al. reported that the nasal side was the thinnest, but they analyzed only the horizontal line through the fovea and did not consider the vertical component. ${ }^{10}$ Park et al. observed that the subfoveal choroidal thickness was significantly greater than the thickness at $1 \mathrm{~mm}$ nasally to the fovea, as well as at 3 $\mathrm{mm}$ nasally, superiorly, and inferiorly to the fovea. ${ }^{12}$ Read et al. described a thinning of the inferior and nasal locations of the macula compared to the superior and temporal locations. ${ }^{11}$ It has been hypothesized that the inferonasal thinning of the choroid may be related to the embryologic closure of the inferior fetal fissure in this general location. ${ }^{18}$ Another possible reason for this observation might be the presence of a watershed zone between the macula and the optic nerve, ${ }^{19}$ observed in $60 \%$ of eyes. ${ }^{20}$ The watershed zone indicates the isolation of the choroidal capillary bed supplied by an independent posterior ciliary artery that does not anastomose with another end artery, making it prone to ischemia and, therefore, to lower choroidal volume.

In our pediatric population, the macular choroidal volume showed a significant negative correlation with AXL after adjustment for age. The significant influence of the AXL on the volume of the choroid also was proven by the use of multiple regression analysis. This result is consistent with the finding of the previous study by Read et al. ${ }^{11}$ However, recently, Park et al. found no correlation between subfoveal choroidal thickness and AXL in children. ${ }^{12}$ This may be related to the smaller number of eyes included in that study compared to the study by Read et al. In adult patients, a negative correlation of choroidal volume with AXL also has been described. ${ }^{4}$ Notably, while in adults the choroid decreases by $0.56 \mathrm{~mm}^{3}$ for every millimeter of AXL, ${ }^{4}$ in our pediatric sample we observed a decrement of $1 \mathrm{~mm}^{3}$ (11.7\%) for every millimeter. This difference may be explained by the fact that the volume of the choroid in children is greater than in adults. So far, to our knowledge no other studies in a pediatric population have reported the quantity of decrement per $\mathrm{mm}^{3}$.

We also found a trend of higher choroidal volume in young females than in young males, with a significance level of 0.056 . Others did not observe any highly significant correlation of choroidal thickness with sex. ${ }^{11,12}$ On the contrary, previous studies in adults showed that the choroid is thicker in men than in women. ${ }^{4,17}$ The significance of this finding remains uncertain; it has been suggested that one biological explanation may be a different hormonal exposure between males and females. Indeed, sex and hormonal status influence the choroidal blood flow, ${ }^{21,22}$ and, therefore, also may influence the choroidal volume. However, as Park et al. have reported recently, the effect of hormones may not be significant, at least in children. ${ }^{12}$ Because of the small difference in choroidal thickness and volume between males and females, our study may be not powered enough to detect real intersexual difference in choroidal volume; therefore, a further study involving a greater number of eyes is needed to answer this question.

We also investigated the influence of other variables, such as height and weight of the children, on the macular choroidal volume. We hypothesized a relationship between the growth of the children's bodies with the growth of the choroid. However, no correlations were found. A larger study is needed to verify our results.

Our study has some limitation. First, it is retrospective in nature; however, we carefully included only children with no ocular or systemic pathologies that are known to affect the choroid. Second, we included only 52 children, which is a relatively small number compared to the large range of age of the subjects (from 2-17 years). Third, we did not consider the effect of other unknown factors in the determination of choroidal volume, such as blood pressure or ocular perfusion pressure. ${ }^{23}$ However, to our knowledge there is no evidence in the literature that they may influence the choroid in the pediatric population. Fourth, the diurnal variability of the choroidal thickness ${ }^{24}$ could have influenced our results; however, all the scans were obtained between 9 AM and 1 PM to minimize this possibility. Finally, the identification of the chorioscleral border was performed manually; however, the reproducibility of this manual technique was demonstrated previously to be very high. Ongoing development of automat-
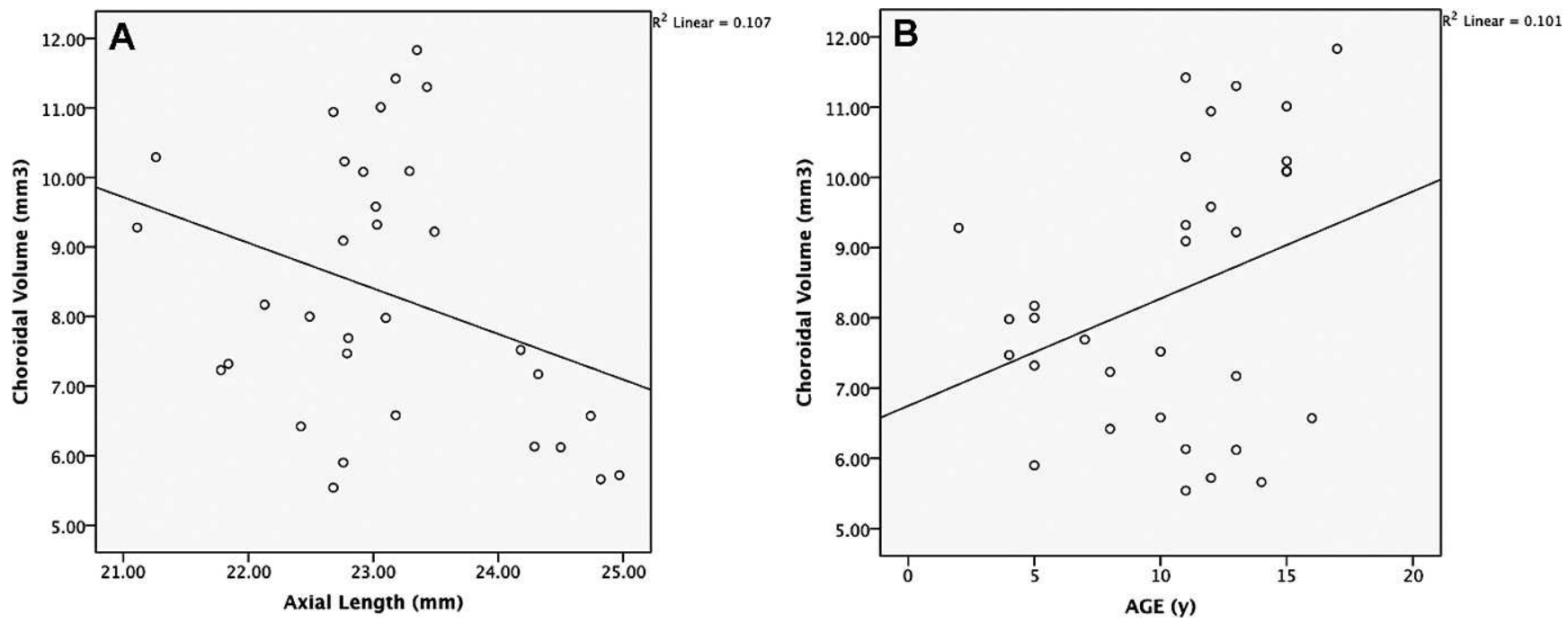

Figure 3. (A) Scatterplot of axial length and macular choroidal volume of all subjects shows a significant negative correlation $\left(P<0.001, R^{2}=\right.$ 0.101). (B) Scatterplot of age and macular choroidal volume of all subjects shows a significant positive correlation $\left(P=0.003, R^{2}=0.101\right)$. 
ed methods of choroidal segmentation will be useful for largescale quantitative studies of the choroid.

In conclusion, the EDI-OCT was a well-tolerated and noninvasive procedure that allowed us to obtain a 3dimensional evaluation of the choroid in children. According to our results, the choroidal volume increases with age during childhood, as do other ocular structures, but decreases with AXL. This finding supports the hypothesis that the choroid grows progressively during childhood. Intersexual differences of choroidal volume also may be present, but further larger studies are needed to clarify this possibility, as well as to elucidate the developmental process of the choroidal tissue and its topographic changes over time.

\section{Acknowledgments}

Disclosure: C. Mapelli, None; L. Dell'Arti, None; G. Barteselli, None; S. Osnaghi, None; E. Tabacchi, None; M. Clerici, None; R. Ratiglia, None; F. Viola, None

\section{References}

1. Linsenmeier RA, Padnick-Silver L. Metabolic dependence of photoreceptors on the choroid in the normal and detached retina. Invest Ophthalmol Vis Sci. 2000;41:3117-3123.

2. Bill A, Sperber G, Ujiie K. Physiology of the choroidal vascular bed. Int Ophthalmol. 1983;6:101-107.

3. Saint-Geniez M, D'Amore PA. Development and pathology of the hyaloid, choroidal and retinal vasculature. Int J Dev Biol. 2004; $48: 1045-1058$.

4. Barteselli G, Chhablani J, El-Emam S, et al. Choroidal volume variations with age, axial length, and sex in healthy subjects: a three-dimensional analysis. Ophthalmology. 2012;119:25722578.

5. Spaide RF, Koizumi H, Pozzoni MC. Enhanced depth imaging spectral-domain optical coherence tomography. Am J Ophthalmol. 2008;146:496-500.

6. Regatieri CV, Branchini L, Fujimoto JG, Duker JS. Choroidal imaging using spectral-domain optical coherence tomography. Retina. 2012;32:865-876.

7. Ellabban AA, Tsujikawa A, Matsumoto A, et al. Macular choroidal thickness measured by swept source optical coherence tomography in eyes with inferior posterior staphyloma. Invest Ophthalmol Vis Sci. 2012;53:7735-7745.

8. Chhablani J, Barteselli G, Wang H, et al. Repeatability and reproducibility of manual choroidal volume measurements using enhanced depth imaging optical coherence tomography. Invest Ophthalmol Vis Sci. 2012;53:2274-2280.

9. Chhablani J, Barteselli G, Bartsch DU, et al. Influence of scanning density on macular choroidal volume measurement using spectral-domain optical coherence tomography. Graefes Arch Clin Exp Ophthalmol. 2013;251:1303-1309.
10. Ruiz-Moreno JM, Flores-Moreno I, Lugo F, Ruiz-Medrano J, Montero JA, Akiba M. Macular choroidal thickness in normal pediatric population measured by swept-source optical coherence tomography. Invest Ophthalmol Vis Sci. 2013;54: 353-359.

11. Read SA, Collins MJ, Vincent SJ, Alonso-Caneiro D. Choroidal thickness in childhood. Invest Ophthalmol Vis Sci. 2013;54: 3586-3593.

12. Park KA, Oh SY. Choroidal thickness in healthy children. Retina. 2013;33:1971-1976.

13. Ikuno Y, Kawaguchi K, Nouchi T, Yasuno Y. Choroidal thickness in healthy Japanese subjects. Invest Ophthalmol Vis Sci. 2010;51:2173-2176.

14. Fujiwara T, Imamura $\mathrm{Y}$, Margolis R, Slakter JS, Spaide RF. Enhanced depth imaging optical coherence tomography of the choroid in highly myopic eyes. Am J Ophthalmol. 2009;148: 445-450.

15. Hirata M, Tsujikawa A, Matsumoto A, et al. Macular choroidal thickness and volume in normal subjects measured by sweptsource optical coherence tomography. Invest Ophthalmol Vis Sci. 2011;52:4971-4978.

16. Song WK, Lee SC, Lee ES, Kim CY, Kim SS. Macular thickness variations with sex, age, and axial length in healthy subjects: a spectral domain-optical coherence tomography study. Invest Ophthalmol Vis Sci. 2010;51:3913-3918.

17. Li XQ, Larsen M, Munch IC. Subfoveal choroidal thickness in relation to sex and axial length in 93 Danish university students. Invest Ophthalmol Vis Sci. 2011;52:8438-8441.

18. Ouyang Y, Heussen FM, Mokwa N, et al. Spatial distribution of posterior pole choroidal thickness by spectral domain optical coherence tomography. Invest Ophthalmol Vis Sci. 2011;52: 7019-7026.

19. Ikuno Y, Tano Y. Retinal and choroidal biometry in highly myopic eyes with spectral-domain optical coherence tomography. Invest Ophthalmol Vis Sci. 2009;50:3876-3880.

20. Hayreh SS. The ophthalmic artery: III. Branches. $\mathrm{Br} J$ Ophthalmol. 1962;46:212-247.

21. Centofanti M, Bonini S, Manni G, Guinetti-Neuschuler C, Bucci MG, Harris A. Do sex and hormonal status influence choroidal circulation? Br J Ophthalmol. 2000;84:786-787.

22. Kavroulaki D, Gugleta K, Kochkorov A, Katamay R, Flammer J, Orgul S. Influence of gender and menopausal status on peripheral and choroidal circulation. Acta Ophthalmol. 2010; 88:850-853.

23. Kim M, Kim SS, Kwon HJ, Koh HJ, Lee SC. Association between choroidal thickness and ocular perfusion pressure in young, healthy subjects: enhanced depth imaging optical coherence tomography study. Invest Ophthalmol Vis Sci. 2012;53:7710-7717.

24. Tan CS, Ouyang Y, Ruiz H, Sadda SR. Diurnal variation of choroidal thickness in normal, healthy subjects measured by spectral domain optical coherence tomography. Invest $O p h$ thalmol Vis Sci. 2012;53:261-266. 